BULL. AUSTRAL. MATH. SOC.

VOL. $22(1980), 259-274$.

\title{
MINIMAL REQUIREMENTS FOR MINKOWSKI'S THEOREM IN THE PLANE I
}

\author{
J.R. Arkinstall
}

\begin{abstract}
Let $K$ be a closed convex set in the Euclidean plane, with area $A(K)$, which contains in its interior only one point 0 of the integer lattice. If $K$ has other than one or three chords bisected by 0 , it is shown that $A(K) \leq 4$. Also, if $K$ has three such chords, $A(K) \leq 4.5$. The results are generalised to any lattice in the plane.
\end{abstract}

\section{Introduction}

Let $\Lambda$ be a lattice in the plane having determinent $\operatorname{det}(\Lambda)$. We say the set $K$ is admissible if it is a closed convex set in the plane with 0 the only point of $\Lambda$ in its interior. Minkowski's fundamental theorem [4] asserts that if an admissible set $K$ is centrally symetric about 0 , then its area $A(K)$ is no greater than $4 \operatorname{det}(\Lambda)$. We call a chord of $K$ which is bisected by 0 , a chord of symmetry of $K$. Minkowski's hypothesis requires that all chords through 0 be chords of symmetry. We say a chord of symmetry of $K$ is extremal if $K$ has parallel supporting lines at its endpoints. Scott [6] has shown $A(K) \leq 4 \operatorname{det}(\Lambda)$ if $K$ has an extremal chord of symmetry. We refer to this as result (1).

Let $s(K)$ denote the number of chords of symmetry of an admissible set $K$. We show

THEOREM 1. If $s(K)$ is even or infinite, $A(K) \leq 4 \operatorname{det}(\Lambda)$.

THEOREM 2. If $s(K)>1, A(K) \leq 4.5 \operatorname{det}(\Lambda)$.

Received 11 April 1980. 
THEOREM 3. If $s(K)>3, A(K) \leq 4 \operatorname{det}(\Lambda)$.

Theorem 2 is analogous to a result of Ehrhart [3], that $A(K) \leq 4.5 \operatorname{det}(\Lambda)$, whenever $K$ satisfies the more restrictive condition that 0 is the centre of gravity of $K$. It will be sufficient to establish the theorems when $\Lambda$ is the integral lattice $\Lambda_{0}$, since $s(K)$ and $A(K) / \operatorname{det}(\Lambda)$ are invariant under a linear transformation of $K$ and $\Lambda$. As $\operatorname{det}\left(\Lambda_{0}\right)=1$, we delete all further reference to $\operatorname{det}(\Lambda)$.

We show that each of the above theorems gives the best possible bound on $A(K)$. Let $E_{1}$ be the convex hull of the points $(-1,2),(2,-1)$ and $(-1,1)$. This triangle $E_{1}$ is admissible, and has only three chords of symmetry, passing through $(1,0),(0,1)$ and $(-1,-1)$ respectively. As $A\left(E_{1}\right)=4.5$ and $s\left(E_{1}\right)=3$, Theorem 2 is best possible.

For Theorems 1 and 3 we argue as follows. Let $U$ be the convex hull of the four points ( $\pm 1, \pm 1)$. Let $C \subseteq U$ be the union of half a regular am-gon and a semicircle on a common diameter with midpoint 0 . Then $s(C)=m>1$. The set $K(\varepsilon)=(1-\varepsilon) U+\varepsilon C, 0<\varepsilon<1$, is an admissible set and since $U$ is centrally symmetric about $0, s(K(\varepsilon))=s(C)=m$. Since for sufficiently small $\varepsilon, A(K(\varepsilon))$ can be arbitrarily close to $A(U)=4$, Theorems 1 and 3 give the best possible bound for finite $s(K)$. Taking $\varepsilon=0$, we obtain the square $U$ with $A(U)=4$ and $s(U)$ infinite.

Finally, let $E_{2}$ be the convex hull of the three points $( \pm t,-1)$ and $(0,1 / t)$ where $t>1$. The triangle $E_{2}$ is admissible, and has only one chord of symmetry parallel to the $x$-axis. As $A\left(E_{2}\right)>t$ and $s\left(E_{2}\right)=1$, we can deduce no upper bound on $A(K)$ from the information that $s(K)=1$.

\section{Proof of Theorem 1}

If all the chords of $K$ which pass through 0 are chords of symmetry of $K$, Minkoswki's theorem gives the desired result. We may thus assume there is a chord of $K, P_{0} O P_{0}^{\prime}$ which is not a chord of symmetry of $K$. 
We denote each chord $P O P^{\prime}$ of $K$ by the label $c(\theta)$, where $\theta$ is the angle $P_{0} O P$ measured in an anti-clockwise direction and $\theta \in[0, \pi]$. The continuous function $d(\theta)=(P O)^{2}-\left(O P^{\prime}\right)^{2}$ has a zero when and only when $c(\theta)$ is a chord of symmetry of $K$, and by our choice of $P_{0} O P_{0}^{\prime}$, $d(0)=-d(\pi) \neq 0$. By applying the intermediate value theorem to $d(\theta)$, we deduce that $d(\theta)$ has at least one zero on $[0, \pi]$. If $s(K)$ is even, then $d(\theta)$ has an even number of zeros, and so at least one zero $\theta_{*}$, occurs at an extremum of $d(\theta)$. Let $\bar{K}$ denote the reflection of $K$ in the origin 0 , and let $P_{*} O P_{*}^{\prime}$ be the chord $c\left(\theta_{*}\right)$. If $d(\theta)$ has a local maximum at $\theta_{*}$, then in a neighbourhood of $P_{*}$ the boundary of $K$ is contained in $\bar{K}^{*}$, and so a supporting line $s$ to $K$ at $P_{*}^{\prime}$, together with a parallel line $s^{\prime}$ through $P_{*}$, form a pair of parallel supporting lines to $K$ at the endpoints of $c\left(\theta_{*}\right)$, a chord of symetry of $K$. We argue similarly if $d(\theta)$ has a local minimum at $\theta_{*}$. In either case, result (1) implies that $A(K) \leq 4$.

If $s(K)$ is infinite, using the above notation, there is a point $A$ on the boundary of $K$, which is an accumulation point of the infinite set $I$ of endpoints $P$ of chords of symmetry. As the boundary of $K$ is continuous, $A$, is itself an endpoint of a chord of symmetry $A Q A^{\prime}$. If we consider a sequence $\left(P_{n}\right)$ in $I$ which has limit $A$, the limit $Z$ of the sequence of lines $\left(P_{n}^{A}\right)$ is a supporting line to $K$ at $A$. Similarly the sequence $\left(P_{n}^{\prime} A^{\prime}\right)$ has as its limit a line $Z^{\prime}$ which is a supporting line to $K$ at $A^{\prime}$. Since, for each $n$, the line $P_{n} A$ is parallel to the line $P_{n}^{\prime} A^{\prime}$, it follows that $Z$ and $Z^{\prime}$ are parallel. Again, result (I) gives that $A(K) \leq 4$, and the proof is complete.

COROLLARY 1. If $d(\theta)$ has a zero at which $d(\theta)$ is an extremu, then $c(\theta)$ is an extremal chord of symmetry, and $A(K) \leq 4$.

\section{Proof of Theorem 2}

By Theorem 1 and its corollary, $A(K) \leq 4$ if either $s(K)=2$, or if any zero of $d(\theta)$ occurs at a goint where $d(\theta)$ is also an extremum. Suppose then that $s(K) \geq 3$. We may assume without loss of generality that $d(\theta)$ is increasing, decreasing and increasing at three consecutive 
zeros $\theta_{1}, \theta_{2}$, and $\theta_{3}$. So $c\left(\theta_{i}\right)=P_{i} 0 P_{i}^{\prime}(1 \leq i \leq 3)$ are chords of symmetry of $K$; let the supporting lines to $K$ at $P_{i}$ and $P_{i}^{\prime}$ meet in $T_{i}$. Our assumption about $d(\theta)$ implies that $T_{1}$ lies to the left of $\overrightarrow{P_{1} P_{1}^{\prime}, T_{2}}$ to the right of $\overrightarrow{P_{2} P_{2}^{\prime}}$ and $T_{3}$ to the left of $\overrightarrow{P_{3} P_{3}^{\prime}}$ as illustrated in Figure 1.

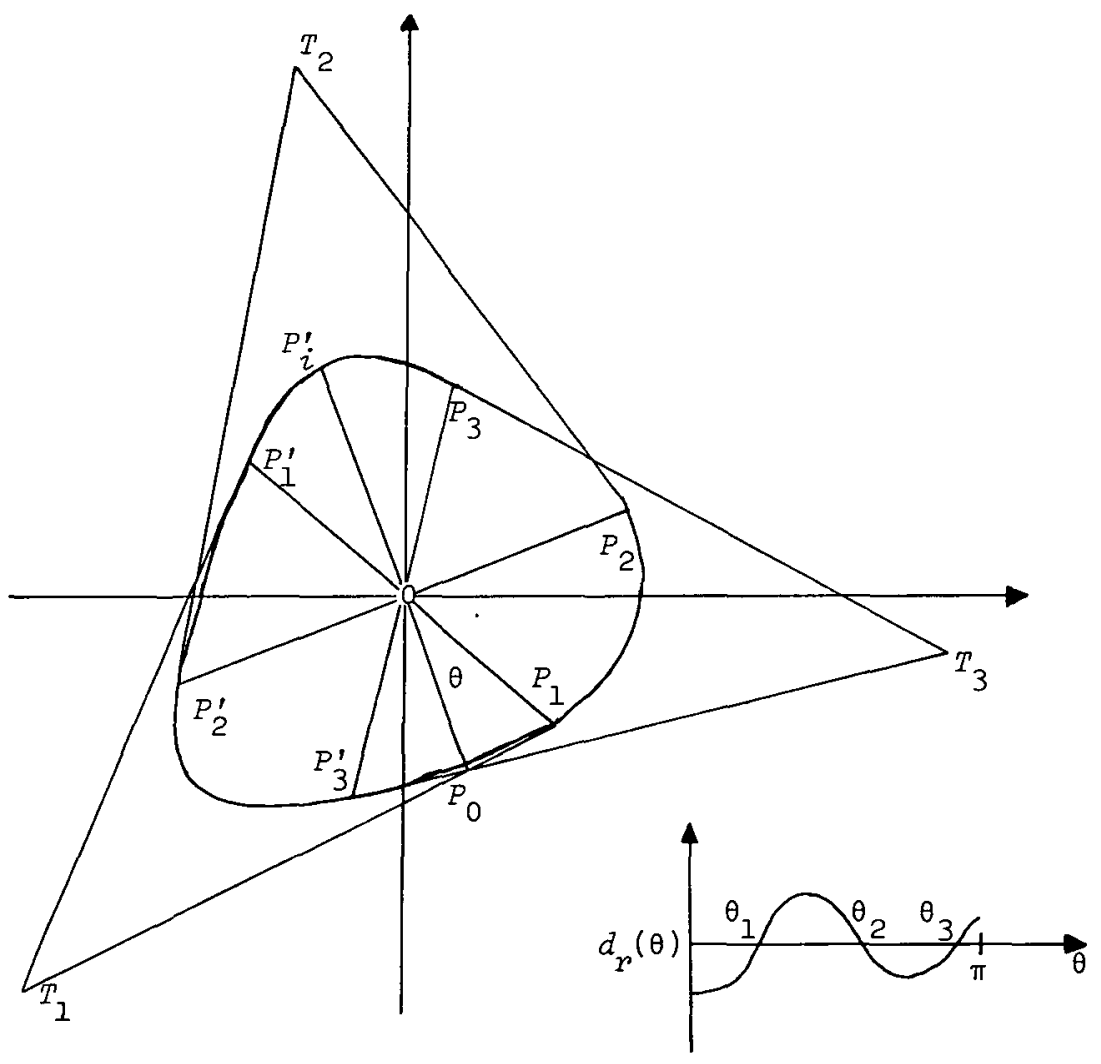

FIGURE 1

Let $K_{i}$ denote the intersection of $K$ with the closed triangular region $P_{i} P_{i}^{\prime} T_{i}(1 \leq i \leq 3)$, and let $\bar{K}_{i}$ be the reflection of $K_{i}$ in 0 . By Minkowski's theorem, as $K_{i} \cup \bar{K}_{i}$ is an admissible set, centraliy symmetric about 0 , 


$$
A\left(K_{i} \cup \bar{K}_{i}\right)=2 A\left(K_{i}\right) \leq 4 \text {. }
$$

It is easily seen that $\bigcup_{i=1}^{3}\left(K \sim K_{i}\right)=\bigcup_{i=1}^{3} K_{i}=K$, and hence that

$\bigcap_{i=1}^{3}\left(K \sim K_{i}\right)=\varnothing$. We now apply a result of Scott [7] which states that if $x_{1}, x_{2}, x_{3}$ are three open convex sets in $E^{2}$, and if $\bigcap_{i=1}^{3} X_{i}=\varnothing$, then

$$
A\left(\bigcup_{i=1} X_{i}\right) \geq 9 / 5 \min _{i=1,2,3} A\left(X_{i}\right)
$$

Taking $X_{i}$ to be the interior of $K \sim K_{i}$, we have

$$
A(K) \geq 9 / 5 \min _{i=1,2,3} A\left(K \sim K_{i}\right) .
$$

However, as $A\left(K_{i}\right) \leq 2(1 \leq i \leq 3)$, we also have

$$
A(K) \leq 2+\min _{i=1,2,3} A\left(K \sim K_{i}\right)
$$

From (2) and (3) we deduce that $A(K) \leq 4.5$; for equality to be attained here, we required equality in both (2) and (3).

Scott [7] shows that equality in (2) is possible only when $K$ is a triangle $T$ with centre of gravity 0 , and with three chords of symmetry, each parallel to an edge of $T$. The set $E_{1}$ of Section 1 is such a triangle.

\section{Polygons and lattice points}

We shall need the following preliminary results. A lattice polygon $P$ is a simple polygon which has each vertex at a lattice point. Let $v, b, c$ denote the number of lattice points which are vertices, boundary points, interior points respectively of $P$, and let $A$ denote the area of $P$. It is known [5] that for lattice $\Lambda_{0}$ and lattice polygon $P$, $A=\frac{2}{2} b+c-1$.

LEMMA 1. Let $P$ be a convex lattice polygon. 
(i) If $v=4$, and $P$ has no pair of parallel edges, then $c \geq 1$. (ii) If $v \geq 5$, then $c \geq 1$.

(iii) If $v=6$, and $c=1$, then $p$ is equivalent under an integral unimodular transformation to the centrally symmetric hexagon $H_{0}$ ilzustrated in Figure 2.

(iv) If $v>6, c \geq 2$.

Proof. Let $P_{1}=V_{1} V_{2} V_{3} V_{4}$ be a convex lattice quadrilateral having no pair of parallel sides and let $P_{2}=V_{1} V_{2} V_{3} V_{4} V_{5}$ be a convex lattice pentagon. In each case, since it is not possible for the sum of every pair of adjacent angles to be less than or equal $\pi$, we may assume that $\underline{I}_{2}+\underline{I V}_{3}>\pi$. Suppose too that $V_{4}$ is no further than $V_{1}$ from the line $V_{2} V_{3}$. Then, in each case, the vertex $X$ of the parallelogram $V_{2} V_{3} V_{4} X$ is a lattice point which is interior to the polygon $P_{i} \cdot$ As any lattice polygon with $v>5$ contains as a subset a lattice pentagon, we have proved (i) and (ii).

Let $L$ be the unique interior lattice point of the convex lattice hexagon $H=V_{1} V_{2} V_{3} V_{4} V_{5} V_{6}$. If $L$ does not lie on $V_{1} V_{4}$, then one of $L V_{4} V_{5} V_{6} V_{1}$ or $L V_{1} V_{2} V_{3} V_{4}$ is a proper convex pentagon, and we deduce from (ii) that $c \geq 2$. Hence $L$ lies on each of the aiagonals $V_{1} V_{4}, V_{2} V_{5}$, $V_{3} V_{6}$. In fact, since the lattice points on a line are regularly spaced, the uniqueness of $L$ implies that $L$ is the comon midpoint of the diagonals. From $(i)$ we deduce that the diagonals are parallel to the edges of $H$, and so $H$ is the image of $H_{0}$ under some integral unimodular transformation.

Finally, let $P$ be a convex lattice polygon with $v \geq 6$ and $c=1$. Choose 5 vertices of $P$. By (iii) each remaining vertex of $P$ lies at the unique lattice point which completes a centrally symmetric hexagon about $L$. Thus $v=6$. Hence if $v>6$ we must have $c \geq 2$. 


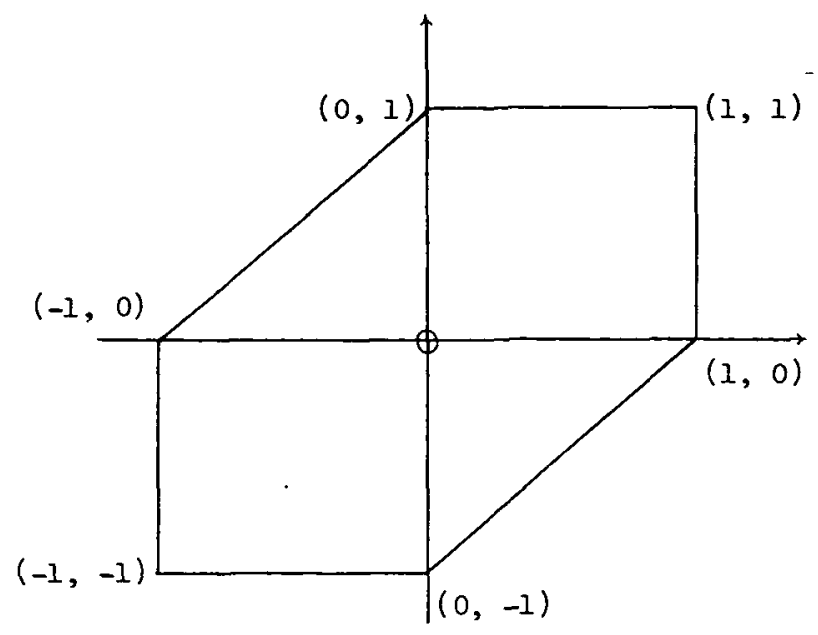

FIGURE 2

LEMMA 2. Let $H_{0}$, with vertices $V_{1} V_{2} V_{3} V_{4} V_{5} V_{6}$ be the convex lattice hexagon of Figure 2. Let $X$, with vertices $T_{1} T_{2} \cdots T_{n}$ be a convex polygon, with $H_{0} \subseteq X$. Let the vertices $V_{2}$ and $V_{3}$ of $H_{0}$ lie on the edge $T_{2} T_{3}$ of $X$, and let the edges $T_{1} T_{2}$ and $T_{3} T_{4}$ of $X$ have $V_{1}$ and $V_{2}$ as midpoints respectively. Then $T_{1}, V_{5}, V_{6}$ and $T_{4}$ are collinear. In particular, if each edge of $X$ contains a vertex of $H_{0}$, $X$ must be a quadrizateral.

Proof. The diagonal $V_{1} V_{4}$ of $H_{0}$ is parallel to, and midway between the edges $V_{2} V_{3}$ and $V_{5} V_{6} \cdot$ Hence $T_{1}$ and $T_{4}$ lie at the same distance from $V_{1} V_{4}$ as $T_{2}$ and $T_{3}$ respectively, that is, on the line $V_{5} V_{6}$.

LEMMA 3. Let $M$ be a polygon having $n$ edges, and having a lattice point at the midpoint of each edge.

(i) If $n$ is odd, each vertex of $M$ is a lattice point.

(ii) If $n$ is even, the lattice polygons with vertices at the alternate midpoints have the same centroid as $M$.

(iii) If $n=4 m+2$ ( $m \in Z)$, and the midpoints of opposite edges occur in pairs which are symmetric about 0 , then $M$ is centrally 
symmetric about 0 .

Proof. Let $v_{i}$ and $\mathrm{m}_{i}$ be the vertices of $M$ and the midpoints of the sides of $M$ respectively, for $i \in I=\{1,2, \ldots, n\}$. With the convention $v_{0}=v_{n}$, we write this as

$$
\frac{3}{2}\left(v_{i-1}+v_{i}\right)=m_{i} \in \Lambda, \quad i \in I \text {. }
$$

When $n$ is odd, equations (4) give

$$
v_{n}=m_{1}-m_{2}+m_{3} \cdots+m_{n} \text {. }
$$

Thus $v_{n} \in \Lambda$, and from (4) we deduce that $v_{i} \in \Lambda$ for all $i \in I$. When $n$ is even, summing the alternate equations in (4) gives

$$
\text { (5) } \quad \frac{1}{2}\left(v_{1}+\ldots+v_{n}\right)=m_{2}+m_{4}+\ldots+m_{n}=m_{1}+m_{3}+\ldots+m_{n-1} \text {. }
$$

We obtain (ii) simply by dividing (5) by $n / 2$.

$$
\text { Now suppose } n=4 m+2 \text {, and } m_{j}=-m_{j+2 m+1} \text {, for }
$$

$j \in J=\{1,2, \ldots, 2 m+1\}$. Adding the expressions in (4) for $\mathrm{m}_{j}$ and $\mathrm{m}_{j+2 m+1}$, we get

$$
\frac{1}{2}\left(v_{j-1}+v_{j}+v_{j+2 m}+v_{j+2 m+1}\right)=0, j \in J .
$$

Taking the alternating sum of these equations gives

$$
\frac{1}{2}\left(v_{4 m+2}+v_{2 m+1}\right)=0 \text {; }
$$

that is $v_{4 m+2}=-v_{2 m+1}$. Using equations (6), it now follows that $M$ is symmetric in 0 .

\section{An analytical result}

In the proof of Theorem 3 we will use two continuous transformations and a limiting process to reduce the number of admissible sets under consideration. We prove

LEMMA 4. Let $\left(K_{i}\right)$ be a sequence of admissible convex sets such that $K_{i} \rightarrow K$ in the Hausdorff metric. Suppose that for alz $i$, $s\left(K_{i}\right)=k$, an odd number, and also that $k_{i}$ has no extremal chord of 
symmetry. Then either

(i) $s(K)=k$, and $K$ has no extremal chord of symmetry, or (ii) $A(K) \leq 4$.

Proof. Let $d(\theta)$ be defined for the set $K$ as in Section 2, with $\theta$ measured from a direction such that $d(0)=-d(\pi) \neq 0$. For all $i$, we define the function $d_{i}(\theta)$ corresponding to the set $K_{i}$ in the same manner, with $\theta$ measured from the same direction used for the set $K$. As no chord of $K_{i}$ is extremal, by Corollary 1 we have that no zero of $d_{i}(\theta)$ is an extremum of $d_{i}(\theta)$. By elementary analysis, we choose an infinite subsequence $\left(K_{i}\right), i \in G \subseteq N$, such that the zeros of $d_{i}(\theta), i \in G$, on the interval $[0, \pi)$, form $k$ convergent subsequences. Since we lose no generality by doing so, we assume that the sequence $\left(K_{i}\right)$ is this subsequence $\left(K_{i}\right), i \in G$. We now show that unless $A(K) \leq 4$, then the limits $\theta_{1}, \theta_{2}, \ldots, \theta_{k}$ of the sequences of zeros of the functions $d_{i}(\theta)$ are all the zeros of $d(\theta)$, and are distinct.

In Section 2 we noted that each function $d_{i}(\theta)$ is continuous in $[0, \pi)$. Since $\left(K_{i}\right)$ converges to $K$ in the Hausdorff metric, the functions $d_{i}(\theta)$ converge to $d(\theta)$ uniformly. Suppose $d\left(\theta_{1}\right) \neq 0$ where $\theta_{1}$ is the limit of a convergent sequence of zeros of $d_{i}(\theta)$. In a neighbourhood of $\theta_{1},|d(\theta)| \geq \frac{1}{2}\left|d\left(\theta_{1}\right)\right|$, by the continuity of $d(\theta)$. Hence, by the uniform convergence of $d_{i}(\theta)$ to $d(\theta)$, there exists a number $N$, so that for $i \geq N,\left|d_{i}(\theta)\right|>0$ for $\theta$ in this neighbourhood of $\theta_{1}$. Therefore no zeros of $d_{i}(\theta)$ lie in this neighbourhood of $\theta_{1}$, for $i \geq N$. By contradiction we have proved that limits of convergent sequences of zeros of $d_{i}(\theta)$ are zeros for $d(\theta)$.

Suppose $\theta_{k+1}$ is a zero of $d(\theta)$ on $[0, \pi)$, not one of the limits above. If $\theta_{k+1}$ is an accumulation point of the set of zeros of $d(\theta)$, then $K$ has an infinite number of chords of symnetry, and Theorem 1 then 
implies $A(K) \leq 4$. If $\theta_{k+1}$ is an extremum of $d(\theta)$, then Corollary 1 implies that $A(K) \leq 4$. We may therefore assume that $\theta_{k+1}$ lies between two neighbourhoods $\left[\theta_{-}, \theta_{K+1}\right)$ and $\left(\theta_{K+1}, \theta_{+}\right]$in which $d(\theta)$ is non zero and of opposite sign. By the uniform convergence of $d_{i}(\theta)$ to $d(\theta)$, there exists a number $N$ such that for $i \geq N, d_{i}(\theta)$ is non zero and of opposite sign in the neighbourhoods $\left[\theta_{-},\left(\theta_{-}+\theta_{K+1}\right) / 2\right]$ and $\left[\left(\theta_{K+1}+\theta_{+}\right) / 2, \theta_{+}\right]$. As each $d_{i}(\theta)$ is a continuous function, for $i \geq N$, $d_{i}(\theta)$ has a zero in the interval $\left(\left(\theta_{-}+\theta_{K+1}\right) / 2,\left(\theta_{K+1}+\theta_{+}\right) / 2\right)$. By again choosing a subsequence of the sequence of functions $\left(d_{i}(\theta)\right)$, we can assert that there is a sequence of zeros of the functions $d_{i}(\theta)$ lying in the above interval, which converge. As shown in the previous paragraph, this sequence converges to a zero of $d(\theta)$ which lies in the above interval, and so must converge to $\theta_{K+1}$. As this contradicts our choice of $\theta_{K+1}$, we deduce that $\theta_{1}, \theta_{2}, \ldots, \theta_{K}$ are the only zeros of $d(\theta)$.

Finally we show that $\theta_{1}, \ldots, \theta_{K}$ are distinct. Suppose to the contrary that $\theta_{1}=\theta_{2}$. Without loss of generality we assume that $\theta_{1}$ and $\theta_{2}$ are the limits of $\left(\theta_{1}(n)\right)$ and $\left(\theta_{2}(n)\right)$ respectively, where $\theta_{1}(n)<\theta_{2}(n)$ are zeros of $d_{n}(\theta)$. For given $n$, and $j \in\{1,2\}$, the zero of $d_{n}(\theta)$ at $\theta_{j}(n)$ corresponds to a chord of symmetry $P_{j, n}{ }^{O P_{j, n}^{\prime}}$ of $k_{n}$. As $n$ becomes large, $P_{1, n}$ and $P_{2, n}$ approach $P$, on the chord of symmetry $P O P^{\prime}$ of $K$ corresponding to the zero of $d(\theta)$ at $\theta_{1}=\theta_{2}$. By again taking a suitable subsequence of $K_{i}$ (if necessary) and renaming this $\left(K_{i}\right)$, we assume that the limit as $n$ becomes large of the angle of orientation of the chord $P_{1, n} P_{2, n}$ exists. We call this limit $\varphi$.

We show that the line $l$ through $P$, with angle of orientation $\varphi$, is a supporting line to $K$ at $P$. For suppose to the contrary that $l$ is a proper chord of $K$ which meets the boundary of $K$ at $Q$. By taking $n$ sufficiently large to make $P_{1, n}$ and $P_{2, n}$ very close to $P$, and the 
angle of orientation of the chord $P_{1, n} P_{2, n}$ very close to $\varphi$, we may assume that the line $P_{1, n_{2, n}}$ passes as close as we choose to $Q$. By convexity $K_{n}$ is bounded by the line $P_{1, n} P_{2, n}$ outside the segment $P_{1, n}{ }^{P}, n$. Thus, taking values of $n$ approaching infinity, and since $K_{n}$ converges to $K, K$ is bounded by the limit of the line $P_{1, \eta,} P_{2, n}$. As this limit is $Z$, we contradict our choice of $Z$ as a proper chord of $K$. Similarly, we can construct a supporting line to $K$ at $P_{*}$ parallel to $l$. By result $(I)$, we then deduce that $A(K) \leq 4$.

\section{Proof of Theorem 3}

Using Minkowski's theorem, Cohn [1] has proved the following boundedness result for convex sets in $E^{d}$. Let $K$ be a convex set which contains in its interior a hypersphere of radius $r$ centred at 0 . If $K$ contains no points of the integer lattice besides 0 in a hypersphere of radius $R=c_{d} / r^{d-1}$, then $K$ contains no lattice points in its interior besides 0 , and in fact lies entirely within the second hypersphere. Cohn establishes a value for the constant $C_{d}$.

We now prove Theorem 3. From Theorem 1 we may assume $s(K)$ is odd, and so at least five. As 0 is an interior point of the convex set $K$, by Cohn [1], $K$ is separated from all non-zero lattice points by a finite number of lines. Hence $K$ is contained in a polygon $K$, bounded by these lines and supporting lines to $K$ at the endpoints of its chords of symmetry. We may clearly choose $K$ so that each edge of $K$ contains in its relative interior at least one endpoint of a chord of symmetry or a lattice point. As $K$ has no smaller area and no fewer lines of symmetry than $K$, we take $K$ to be the polygon $K$. Should an edge of $K$ contain an endpoint of each of three or more chords of symmetry, by the convexity of $K$ the other endpoints lie on a parallel edge. From result (1), we then know that $A(K) \leq 4$. We therefore may assume that the polygon $K$ has at least five edges.

We now modify the polygon $K$ so that each edge of $K$ contains at least one lattice point in its relative interior. If an edge $E$ of $K$ contains no lattice point in its relative interior, we form edge $E(t)$, 
parallel to $E$ and distance $t$ further than $E$ away from 0 . Denote by $K(t)$ the polygon obtained from $K$ by including this new edge and extensions of the edges of $K$ adjacent to it. We continue to increase $t$ until one of three things happens:

(a) the length of $E(t)$ becomes zero, or

(b) $E(t)$ has a lattice point on it, in its relative interior, or

(c) $s(K(t)) \neq s(K)$.

One of these things must happen, since as $K$ contains a disc about 0 , each modified set $K(t)$ also contains the same disc, and, by Cohn [1], such sets are uniformly bounded.

Suppose (c) occurs either with or before either (a) or (b). This can occur in two ways. Firstly, we suppose that $k\left(t^{*}\right)$ is the first modified set for which $s(K) \neq s\left(K\left(t^{*}\right)\right)$. As neither (a) or (b) are true for $t<t^{*}$, each of the sets $K_{i}=K\left(t^{*} \cdot(1-1 / i)\right)$ is admissible, and since (c) is false also for $t<t^{*}, s\left(K_{i}\right)=s(K)$ is odd for each set. As the sequence $\left(K_{i}\right)$ converges to $K\left(t^{*}\right)$ in the Hausdorff metric, we can deduce from Lemma 4 that $A\left(K\left(t^{*}\right)\right) \leq 4$.

Secondly, we suppose that $s(K)=s\left(K\left(t^{*}\right)\right)$ for all $t \in\left[0, t^{*}\right]$, but that this equality holds on no longer interval. By our assumption that ( $c$ ) occurs with or before (a) or (b), and noting that the transformation is such that (a) or (b) must occur for some first modified set, we may assume that neither (a) nor (b) occur for $t<t^{*}+\delta$, for some $\delta>0$. Thus we can choose a sequence $K_{i}=K(t), t \in\left(t^{*}, t^{*}+\delta .1 / i\right)$, of admissible sets, such that $\left(K_{i}\right)$ converges to $K\left(t^{*}\right)$ in the Hausdorff metric. We assume that $s\left(K_{i}\right)$ is odd and finite for each $i$, since otherwise we could deduce by Theorem 1 that $A\left(K_{i}\right)$, and so $A\left(K\left(t^{*}\right)\right)$, is no greater that 4 . In fact, as only three edges of $K(t)$ are modified by the transformation, the value of $s\left(K_{i}\right)$ differs from $s\left(K\left(t^{*}\right)\right)$ by at most six, for we showed in an earlier argument that $A(K(t)) \leq 4$ if any edge of $K(t)$ contains the endpoints of more than two chords of symmetry. Hence we can choose a subsequence of $K_{i}$, which we relabel $\left(K_{i}\right)$, so that each $s\left(K_{i}\right)$ is odd, constant, and not equal to $s\left(K\left(t^{*}\right)\right)$. By 
applying Lemma 4 to this sequence $\left(K_{i}\right)$, we deduce that $A\left(K\left(t^{*}\right)\right) \leq 4$.

We may therefore assume that $K$ has at least five edges, each of which contains a lattice point in its relative interior. By Lemma 1 ( $i i$ ), we deduce that 0 is an interior point of the lattice polygon $Y$ whose vertices are those lattice points on the boundary of $K$.

We now further modify $K$ so that each edge $E$ of $K$ either contains two lattice points in its relative interior, or one lattice point at its midpoint. We show that such a modification increases the area $A(K)$, does not decrease $s(K)$ and does not remove from the boundary of $K$ any of the lattice points on it. Suppose $L$, not the midpoint of edge $E$ of $K$, is the only lattice point in the relative interior of $E$. We replace $E$ by another edge $E(\psi)$, through $L$, at angle $\psi$ to $E$. As $L$ is not the midpoint of $E$, we may orient $\psi$ so that for (small) positive $\psi$, the set $K(\psi)$ bounded by $E(\psi)$ and the extensions of edges of $K$ other than $E$, has area greater than $A(K)$. As the boundary of $K$ is continuous, and since $E(\psi)$ pivots about $L$, which is not its midpoint, $A(K(\psi))$ increases continuously with $\psi$ until one of three things happens:

(d) $L$ is the midpoint of $E(\psi)$, or

(e) $E(\psi)$ contains two lattice points in its relative interior, or

(f) $s(K(\psi)) \neq s(K)$.

No lattice points are lost from the boundary of $K$ this modification of $K$. For, were $L_{1}$ to be lost at angle $\psi$, as $L$ is not the midpoint of $E(\psi)$, the lattice point $2 L-L_{1}$ lies in the relative interior of $E(\psi)$. Hence the convex hull of those lattice points on the boundary of $K, Y \subseteq K(\psi)$. As 0 is an interior point of $Y$, we have by Cohn [1] that the sets $K(\psi)$ are uniformly bounded. Hence, for sufficiently large $\psi,(d)$ or (e) must occur. Suppose (f) occurs either with or before either $(d)$ or $(e)$.

This can occur in two ways. Firstly suppose $K\left(\psi^{*}\right)$ is the first modified set for which $s\left(K\left(\psi^{*}\right)\right) \neq s(K)$. The sequence of admissible sets $K_{i}=K\left(\psi^{*}(1-1 / i)\right)$ converges to $K\left(\psi^{*}\right)$ in the Hausdorff metric, and $s\left(K_{i}\right)=s(K)$ is odd for each set $K_{i}$ in the sequence. By applying Lemma 4 to the sequence $\left(K_{i}\right)$ we deduce that $A\left(K\left(\psi^{*}\right)\right) \leq 4$. 
Secondly, suppose that $s(K)=s(K(\psi))$ for al]. $\psi \in\left[0, \psi^{*}\right]$, but this equality holds on no longer interval. We can apply the same argument used above for $K(t)$, noting carefully that only three edges of $K$ are modified by this transformation on any sufficiently small open interval. As above we can deduce that $A\left(K\left(\psi^{*}\right)\right) \leq 4$ in this case.

In performing the above modifications to the polygon $K$, there is a possibility that an infinite sequence of modifications may occur. For example, a sequence of edge turning modifications may successively upset the previously set lattice point midpoints of edges. However, all the modifications of $K$ contain $Y$, and so contain a fixed disc about 0 . By Cohn [1], all such modifications of $K$ are bounded by a uniform bound.

We may thus apply Blaschke's selection theorem [2] to any such sequence of modifications of $K,\left(K_{i}\right)$, and obtain a limiting figure $K^{*}$, which can no longer be modified. However, $s\left(K_{i}\right)=s(K)$ is odd for each of these admissible sets $K_{i}$, and the sequence $K_{i}$ converges to $K^{*}$ in the Hausdorff metric. Hence by Lemma 4 we deduce that unless $s\left(K^{*}\right)=s(K)$ then $A(K) \leq 4$.

We therefore may assume $s\left(K^{*}\right)=s(K)$, and since $K^{*}$ is immutable by the above modifications it must be a convex polygon, with at least five edges, each edge containing at least one lattice point in its relative interior. Further, if any edge $E$ of $K^{*}$ contains only one lattice point $L$ in its relative interior, $L$ is the midpoint of $E$. We call such an edge $E$ a lattice midpoint edge. Since $s\left(K^{*}\right)=s(K) \geq 5$, by Theorem 2 we deduce that $A\left(K^{*}\right) \leq 4.5$.

Denote by $Z$ the convex lattice polygon formed by the convex hull of the set of lattice points which lie on the boundary of $K^{*}$, but are not vertices of $K^{*}$. As 0 is the only possible lattice point in the interior of $Z \subseteq K^{*}$, we deduce from Lemma $1(i v)$ that $K^{*}$ has either,

(g) six edges, each lattice midpoint edges, or

(h) five edges, one containing two lattice points in its relative interior, the other four edges each lattice midpoint edges, or

(i) five edges, each lattice midpoint edges. 
We first show that cases $(g)$ and $(h)$ cannot arise. In case $(g)$, by Lemma I ( $i$ ii), $Z$, having six lattice points on its boundary, is a centrally symmetric hexagon $H$ which is an affine transform of $H_{0}$, the hexagon shown in Figure 2. The incidences of $K^{*}$ with $H$ listed in $(g)$, become, when transformed by the inverse of this affine transformation, incidences of the image of $K^{*}$ with $H_{0}$. Indeed, these incidences are exactly those needed for Lemma 2. Hence the image of $K^{*}$, and hence $K^{*}$, is a quadrilateral. Hence case $(g)$ cannot occur. In case $(h)$, by Lemma 3 (i), each vertex of $K^{*}$ is also a lattice point, and so $K^{*}$ has ten boundary lattice points. By Pick's theorem, we deduce that $A\left(K^{\star}\right)=5$. However, since this contradicts the bound on $A\left(K^{*}\right)$ given above by Theorem 2 , case (h) cannot arise.

Finally, in case ( $i$ ), by Lemma 1 ( $i i i)$, as $Z$ has six vertices and only one interior lattice point, it is a centrally symmetric hexagon. Thus the lattice points on the boundary of $K^{*}$ number 6 , and are symmetrically located about 0 . By Lemma 3 ( $i i i$ ) then, $K^{*}$ is itself centrally symmetric about 0 . By Minkowski's theorem, we deduce that $A\left(K^{*}\right) \leq 4$.

\section{References}

[1] Harvey Cohn, "On finiteness conditions for a convex body", Proc. Amer. Math. Soc. 2 (1951), 544-546.

[2] H.G. Eggleston, Convexity (Cambridge Tracts in Mathematics and Mathematical Physics, 47. Cambridge University Press, Cambridge, 1958).

[3] Eugene Ehrhart, "Une Generalisation du theoreme de Minkowski", C.R. Acad. Sci. Paris Ser. A 240 (1955), 483-485.

[4] Hermann Minkowski, Geometrie der Zahlen (Druck und Teubner, Leipzig und Berlin, 1910; reprinted Chelsea, New York, 1953).

[5] G. Pick, "Geometrisches zur Zahlenlehre", Sitzungsber. Lotos Prag. (2) $19(1900), 311-319$.

[6] P.R. Scott, "Convex bodies and lattice points", Math. Mag. 48 (1975), $110-112$. 
[7] P.R. Scott, "On three sets with no point in common", Mathematika 25 (1978), 17-23.

Department of Pure Mathematics, University of Adelaide,

Adelaide,

South Australia 5001,

Austral ia. 